\title{
Association of sleep duration and insulin resistance in Taiwanese vegetarians
}

\author{
Jiunn-Kae Chang ${ }^{1}$, Malcolm Koo², Vivia Yu-Ying Kao ${ }^{1}$ and Jui-Kun Chiang ${ }^{1,3^{*}}$
}

\begin{abstract}
Background: Short sleep duration has been reported to associate with increased insulin resistance. However, no studies have investigated whether such association exists in vegetarians. The aim of this study was to investigate the association between sleep duration and insulin resistance in Taiwanese vegetarians.

Methods: A total of 1290 individuals were recruited from a regional hospital in south Taiwan during their regular routine physical examination. Only individuals who described themselves as Buddhist vegetarians were included in the study. Demographic information and clinical characteristics were collected and multiple logistic regression analysis was used to evaluate the association between sleep duration and insulin resistance.

Results: A total of 433 vegetarians were included in the study. Results from univariate logistic regression indicated that insulin resistance was significantly associated with male sex, greater waist circumference, higher triglyceride levels, lower high-density lipoprotein cholesterol levels, higher plasma creatinine levels, higher alanine transaminase levels, greater energy expenditure, and sleep duration of more than 8 hours per night. Multiple logistic regression revealed that insulin resistance was significantly and independently associated with sleep duration of more than 8 hours per night (odd ratios $=2.27,95 \%$ confidence interval $=1.24,4.11$ ) after adjusting for waist circumference and levels of alanine transaminase.
\end{abstract}

Conclusions: Sleep duration of more than 8 hours per night is an independent risk factor associated with increased insulin resistance in vegetarians.

Keywords: Sleep duration, Insulin resistance, Vegetarian

\section{Background}

The marked reduction in average sleep duration in modern society has been suggested as one of the risk factors responsible for the increase in prevalence of metabolic syndrome, diabetes, and obesity [1-3]. Short sleep duration has been shown to independently associate with weight gain [4] and an increased risk of hypertension [5], cardiovascular disease [6], and colorectal adenoma [7]. In addition, a U-shaped relationship between sleep duration and hyperglycemia [8], metabolic syndrome [9], diabetes [10,11], and even mortality [12] has been reported. Recently, an association between short sleep

\footnotetext{
*Correspondence: jkch68@gmail.com

'Department of Biotechnology, Chia Nan University of Pharmacy \& Science, Tainan, Taiwan

${ }^{3}$ Department of Family Medicine, Buddhist Dalin Tzu Chi General Hospital, 2 Min-Sheng Road, Dalin, Chiayi, Taiwan

Full list of author information is available at the end of the article
}

duration and increased insulin resistance was noted $[13,14]$. It has been postulated that decreased sleep duration could elevate appetite, increase body weight, decrease glucose tolerance, increase blood pressure, and increase sympathetic tone $[15,16]$. Insulin resistance is defined as the decreased tissue response to insulinmediated cellular actions. It is the main pathophysiological feature of the metabolic syndrome, which is strongly associated with a number of health problems such as diabetes, cardiovascular disease, hypertension, and hyperlipidaemia [17-20].

A few studies have reported the beneficial effects of vegetarian dietary patterns on the prevention of metabolic syndrome. Foods with a low glycemic index and high dietary fiber content typically consumed by vegetarians are associated with a lesser increase in post-prandial blood glucose and insulin secretion [21]. In a crosssectional survey of a representative sample of 19,003

\section{Biomed Central}


adults in a suburban area of Beijing, vegetarians had a lower risk of metabolic syndrome [22]. Similar results where a vegetarian dietary pattern is associating with a lower risk of metabolic syndrome were also observed in a cross-sectional study of 773 adults randomly selected from the Adventist Health Study 2. The relationship persisted after controlling for the possible confounding effects of age, sex, ethnicity, smoking, alcohol intake, physical activity, and dietary energy intake [23]. In addition, a study on female Taiwanese reported that serum total cholesterol, LDL-cholesterol, triglycerides, fasting blood glucose, and blood pressure were significantly lower in long-term vegetarians compared with omnivores [24]. However, it is not known whether the association between sleep duration and insulin resistance also exists in vegetarians who are potentially less prone to insulin resistance compared to omnivores. The aims of this study were to investigate the association between sleep duration and insulin resistance in Taiwanese vegetarians and examine whether such association is independent of adiposity. The area under the receiver operating characteristic curve was used to quantify prediction accuracy.

\section{Methods}

\section{Study participants}

Participants were recruited from a regional hospital in south Taiwan at the time of their regular routine physical examination between May 2007 and April 2009. Demographic information and clinical characteristics including age, sex, body weight, height, waist circumference, a family history of diabetes or hypertension, dietary habit, sleep duration, and sitting blood pressure were collected. Individuals with diabetes, cancers, and chronic renal failure on hemodialysis were excluded from the study.

In addition, only individuals who described themselves as Buddhist vegetarians were included in the study. Chinese Buddhist vegetarians avoid all meat and fish consumption in their diet. Other than that, their diet is similar to the usual Chinese diet in terms of cooking methods, choice of staple (primarily rice), and consumption of fruits and vegetables. Some Chinese Buddhist vegetarians also include eggs and dairy products in their diets [25]. Results from a nutritional study on 109 Taiwanese vegetarians and 107 age- and sex-matched omnivores found that energy intake was significantly lower in female vegetarians but not in male vegetarians when compared with the omnivore counterparts. Vegetarians also consumed significantly less protein, fat, and cholesterol than the omnivores [26]. Another study on 49 healthy Buddhist lactovegetarians and 49 omnivores in Taiwan also reported that vegetarians consumed less energy, fat, and protein, but more dietary fiber than the omnivores [27].
Body weight and height were measured while all participants were minimally clothed without shoes using digital scales. Waist circumference was obtained at the umbilical level using a measuring tape. Body mass index was computed as the ratio of weight (in kilograms) to height (in meters) squared.

Sleep duration and energy expenditure were assessed using a questionnaire administered face-to-face by a research assistant. Mean sleep duration was calculated as $[(5 / 7 \times$ weekday sleep duration $)+(2 / 7 \times$ weekend sleep duration)] [28]. Daily energy expenditure was estimated using a seven-day physical activity recall questionnaire (Chinese version) [29]. The study protocol was approved by the Research Ethics Committee of the Buddhist Dalin Tzu Chi General Hospital (Approved No. B09702038, B09604003-1) and all participants provided written informed consent.

\section{Sample processing and analyses}

Total cholesterol, triglyceride, high-density lipoprotein cholesterol (HDL-C), low-density lipoprotein cholesterol (LDL-C), glucose, white blood cell counts, blood urea nitrogen (BUN), creatinine, insulin, and alanine transaminase (ALT) were measured using blood sample collected from each participants after a minimum eight hour fasting period. The tests were analyzed using an auto-analyzer (Sysmex XE-2100 Blood Cell Analyzer, Kobe, Japan) at the central laboratory of the study hospital. Plasma fasting insulin concentrations were measured using human insulin Enzyme Linked Immunosorbent Assay (ELISA) kit (BioSource Europe S.A., Nivelles, Belgium). Insulin resistance was defined by homeostasis model assessment for insulin resistance (HOMA-IR). HOMA-IR was calculated by dividing the product of fasting plasma glucose $(\mathrm{mg} / \mathrm{dL})$ and fasting plasma insulin (mU/L) by 405 [30]. Insulin resistance was defined as the value of HOMA-IR of highest quartile of its empirical distribution [31], which is $\geq 2$ in this study.

\section{Statistical analysis}

Sleep duration was dichotomized using a cut off value of 8 hours or less in all analyses. Continuous variables were expressed as mean \pm standard deviation (SD). Categorical variables were represented by frequency and percentage. In the univariate analysis, Student $t$-tests or Wilcoxon rank-sum tests were used to compare differences between the two groups, depending on the results of Shapiro-Wilk test of normality. Fisher's exact tests were used to compare the categorical variables between the two groups.

Univariate logistic regression analysis was performed for the variables on demographic and clinical characteristics with the binary variable insulin resistance as the 
dependent variable. Multiple logistic regression analysis with stepwise variable selection procedure using the Akaike's Information Criterion (AIC) was conducted to compute adjusted odds ratios (ORs) and 95\% confidence intervals (CI)s for the association of sleep duration with insulin resistance. Variables that were under evaluation during the stepwise model development included sex, age, waist circumference, white blood cell count, total cholesterol, triglyceride, HDL-C, LDL-C, creatinine, blood urea nitrogen, ALT, education level, energy expenditure, and sleep duration. Hosmer and Lemeshow Goodness-of-Fit test was conducted to determine the adequacy of the fitted logistic models.

A receiver operating characteristic (ROC) analysis was conducted to evaluate the ability for correctly discriminating the study participants of low and elevated HOMA-
IR. Plots of the sensitivity (true-positive fraction) versus 1 - specificity (false-positive fraction) were made and the overall diagnostic accuracy was quantified using the area under the curve (AUC). All analyses were performed using the statistics package R 2.13.0 [32] and a 2-sided $\mathrm{P}<0.05$ was considered statistically significant.

\section{Results}

A total of 1,290 individuals were approached at their physical examination. Of those, 515 were identified as vegetarians and 485 (94.2\%) agreed to participate in the study. A further 35 individuals were excluded because of their diseases and 17 individuals had missing data. The basic characteristics of the 433 study participants are shown in Table 1. Individuals with sleep duration of more than 8 hours had significantly greater waist circumference, energy

Table 1 Characteristics of the study participants according to sleep duration

\begin{tabular}{|c|c|c|c|}
\hline \multirow[t]{2}{*}{ Variable } & \multicolumn{2}{|c|}{ Sleep duration } & \multirow[t]{2}{*}{$\mathbf{P}$} \\
\hline & $\leq 8$ hours, $n=371$ & $>8$ hours, $n=62$ & \\
\hline Sex & & & $<0.001$ \\
\hline Male & $77(20.8)$ & $30(48.4)$ & \\
\hline Female & $294(79.2)$ & $32(51.6)$ & \\
\hline Age (years) & $55.9 \pm 9.0$ & $63.7 \pm 8.3$ & 0.079 \\
\hline Body mass index $\left(\mathrm{kg} / \mathrm{m}^{2}\right)$ & $22.9 \pm 2.8$ & $23.2 \pm 2.3$ & 0.238 \\
\hline Waist circumference $(\mathrm{cm})$ & $74.0 \pm 7.8$ & $77.2 \pm 7.9$ & 0.002 \\
\hline Systolic blood pressure $(\mathrm{mmHg})$ & $125.3 \pm 19.1$ & $124.2 \pm 19.7$ & 0.914 \\
\hline Education & & & 0.033 \\
\hline Primary school and below & $112(32.1)$ & $11(18.3)$ & \\
\hline High school and above & $237(67.9)$ & $49(81.7)$ & \\
\hline Alcohol use & $4(1.1)$ & 0 & 1.000 \\
\hline Smoking & $1(0.3)$ & 0 & 1.000 \\
\hline Energy expenditure (Kcal $\times 10^{3} /$ day) & $2.7 \pm 1.0$ & $3.2 \pm 1.3$ & 0.034 \\
\hline Family history of hypertension & $58(15.6)$ & $8(12.9)$ & 0.704 \\
\hline Family history of diabetes & $90(24.3)$ & $17(27.4)$ & 0.634 \\
\hline White blood cell count $\left(\times 10^{3} / \mu \mathrm{L}\right)$ & $6.0 \pm 1.6$ & $6.6 \pm 1.6$ & 0.014 \\
\hline Total cholesterol (mmol/L) & $4.6 \pm 0.8$ & $4.5 \pm 0.7$ & 0.303 \\
\hline Triglyceride $(\mathrm{mmol} / \mathrm{L})$ & $1.2 \pm 0.7$ & $1.5 \pm 0.8$ & 0.001 \\
\hline $\mathrm{HDL}-\mathrm{C}(\mathrm{mmol} / \mathrm{L})$ & $1.42 \pm 0.36$ & $1.18 \pm 0.25$ & $<0.001$ \\
\hline $\mathrm{LDL}-\mathrm{C}(\mathrm{mmol} / \mathrm{L})$ & $2.98 \pm 0.74$ & $2.95 \pm 0.60$ & 0.902 \\
\hline $\mathrm{ALT}(\mathrm{IU} / \mathrm{L})$ & $22.1 \pm 18.6$ & $25.4 \pm 17.8$ & 0.080 \\
\hline Blood urea nitrogen $(\mathrm{mmol} / \mathrm{L})$ & $4.7 \pm 1.3$ & $4.4 \pm 1.2$ & 0.393 \\
\hline Creatinine $(\mu \mathrm{mol} / \mathrm{L})$ & $56.3 \pm 17.7$ & $60.7 \pm 16.2$ & 0.013 \\
\hline Fasting glucose (mmol/L) & $4.88 \pm 0.49$ & $5.02 \pm 0.47$ & 0.037 \\
\hline Insulin (mU/L) & $6.2 \pm 5.7$ & $7.9 \pm 6.4$ & 0.020 \\
\hline HOMA-IR & $1.4 \pm 1.3$ & $1.8 \pm 1.4$ & 0.010 \\
\hline
\end{tabular}

Continuous and categorical variables were expressed as mean \pm standard deviation and number (percentage).

HDL-C: high-density lipoprotein cholesterol.

LDL-C: low-density lipoprotein cholesterol.

ALT: alanine transaminase. 
Table 2 Results of univariate logistic regression associated with insulin resistance

\begin{tabular}{|c|c|c|c|c|}
\hline & $\beta$ & Standard Error & $\mathbf{P}$ & Odds Ratio (95\% Confidence Interval) \\
\hline Sex (male vs. female) & -1.51 & 0.14 & $<0.001$ & $2.20(1.34-3.59)$ \\
\hline Age & -0.01 & 0.01 & 0.928 & $1.00(0.97-1.02)$ \\
\hline Waist circumference $(\mathrm{cm})$ & 0.07 & 0.01 & $<0.001$ & $1.07(1.04-1.11)$ \\
\hline White blood cell count $\left(\times 10^{3} / \mu \mathrm{L}\right)$ & 0.13 & 0.04 & 0.066 & $1.14(0.99-1.31)$ \\
\hline Total cholesterol (mmol/L) & -0.13 & 0.14 & 0.347 & $0.87(0.66-1.15)$ \\
\hline Triglyceride $(\mathrm{mmol} / \mathrm{L})$ & 0.35 & 0.14 & 0.016 & $1.42(1.07-1.89)$ \\
\hline $\mathrm{HDL}-\mathrm{C}(\mathrm{mmol} / \mathrm{L})$ & -1.25 & 0.38 & 0.001 & $0.29(0.13-0.59)$ \\
\hline $\mathrm{LDL}-\mathrm{C}(\mathrm{mmol} / \mathrm{L})$ & -0.09 & 0.16 & 0.595 & $0.92(0.66-1.26)$ \\
\hline Creatinine $(\mu \mathrm{mol} / \mathrm{L})$ & 0.02 & 0.01 & 0.005 & $1.02(1.01-1.03)$ \\
\hline Blood urea nitrogen $(\mathrm{mmol} / \mathrm{L})$ & 0.09 & 0.09 & 0.315 & $1.09(0.92-1.29)$ \\
\hline $\mathrm{ALT}(\mathrm{IU} / \mathrm{L})$ & 0.02 & 0.01 & 0.002 & $1.02(1.01-1.04)$ \\
\hline Education (primary school and below vs. above) & -0.40 & 0.28 & 0.153 & $0.67(0.38-1.14)$ \\
\hline Energy expenditure $\left(\mathrm{Kcal} \times 10^{3} /\right.$ day $)$ & 0.28 & 0.10 & 0.008 & $1.32(1.07-1.62)$ \\
\hline Sleep duration (>8 hrs vs. $\leq 8$ hrs) & 1.00 & 0.29 & 0.001 & $2.72(1.52-4.80)$ \\
\hline
\end{tabular}

Reference category for education was primary school and below.

HDL-C: high-density lipoprotein cholesterol.

LDL-C: low-density lipoprotein cholesterol.

ALT: alanine transaminase.

expenditure, white blood cell count, triglyceride, plasma creatinine, fasting glucose, insulin, and HOMA-IR but lower HDL-C than those with sleep duration of 8 hours or less. Male participants and those with education levels of high school and above were also significantly associated with sleep duration of more than 8 hours.

Univariate logistic regression analysis revealed that females, greater waist circumference, higher triglycerides, higher creatinine, higher ALT, higher energy expenditure, lower HDL-C, and sleep duration of more than 8 hours were associated with increased risk of insulin resistance (Table 2). Table 3 shows the final model for the predictors in the multivariate logistic regression analysis. The risk of insulin resistance in individuals with sleep duration of more than 8 hours was 2.3 times higher than those with shorter sleep duration, after adjusted for waist circumference and ALT level. The Hosmer-Lemeshow test showed a good fit $(\mathrm{P}=0.259)$. The AUC for the prediction model was 0.69 (Figure 1). The programming code for calculating the probability of insulin resistance based on the final model is provided in Additional file 1: Appendix 1.

An additional multivariate logistic regression analysis was conducted with tertiles of sleep duration. With adjustment of waist circumference and ALT level, short sleep duration ( $<6$ hours), compared to sleep duration of 6 to 8 hours, was not significantly associated with insulin resistance $(P=0.721)$. However, long sleep duration ( $>8$ hours), compared to sleep duration of 6 to 8 hours, was significantly associated with insulin resistance $(P=0.012)$. Therefore, a U-shaped pattern between sleep duration and insulin resistance was not observed in the present study. The choice of cutpoints for sleep duration was based on a plot of nonparametric smoothing obtained from the generalized additive model of sleep duration (Figure 2).

\section{Discussion}

The present study, to the best of our knowledge, is the first to report that sleep duration of 8 hours or more is

Table 3 Results of multiple logistic regression analysis associated with insulin resistance

\begin{tabular}{lcccc}
\hline & $\boldsymbol{\beta}$ & Standard Error & $\mathbf{P}$ & Odds Ratio (95\% Confidence Interval) \\
\hline Intercept & -6.43 & 1.17 & $<0.001$ & \\
Sleep duration (>8 hrs vs. $\leq 8$ hrs) & 0.82 & 0.31 & 0.007 & $2.27(1.24-4.11)$ \\
Waist circumference (cm) & 0.06 & 0.02 & $<0.001$ & $1.06(1.03-1.10)$ \\
ALT (IU/L) & 0.01 & 0.01 & 0.023 & $1.01(1.00-1.03)$ \\
\hline
\end{tabular}

ALT: alanine transaminase.

Variables eliminated during the stepwise regression process included sex, age, white blood cell count, total cholesterol, triglyceride, high-density lipoprotein cholesterol, low-density lipoprotein cholesterol, creatinine, blood urea nitrogen, education level, and energy expenditure.

Akaike's Information Criterion (AIC): 423.5.

The Hosmer-Lemeshow test, $\mathrm{P}=0.259$.

Nagelkerke $\mathrm{R}^{2}=0.13$. 


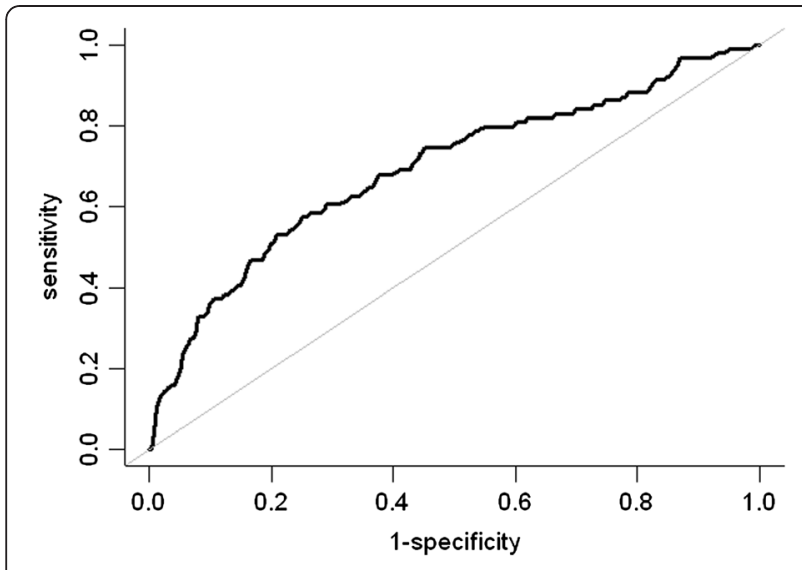

Figure 1 The receiver operating characteristics curve. Model was obtained from the multivariate logistic regression with the area under the curve $=0.69$.

associated with increased risk of insulin resistance in vegetarians. The association remained significant after adjustment for waist circumference and liver function. Previous reports on sleep duration and insulin resistance or metabolic syndrome have indicated the existence of either an inverse [13,14] or a U-shaped relationship [9]. Short sleep duration has been suggested to associate with an increase risk of metabolic syndrome through an upregulation of the neuroendocrine control of appetite, in particular, reduced leptin and increased ghrelin levels. These endocrine changes could lead to weight gain and a higher risk of obesity [33]. Short sleep duration can increase cortisol concentrations and sympathetic nervous system activity, which are associated with increased risk of developing insulin resistance [34].

On the other hand, there is no clear mechanism to explain the association between long sleep duration and insulin resistance. It has been hypothesized that long sleep duration is an indication of the presence of comorbidity such as depression [35]. In addition, low level of physical activity [36], and low socioeconomic status [35] have been reported to associate with long duration of sleep. In the present study, education levels and energy expenditure were not significantly associated with long sleep duration. Another possible mechanism for linking sleep duration with insulin resistance is through the mediation of cytokines. It has been shown that each additional hour of habitual sleep duration was significantly associated with an $8 \%$ increase in C-reactive protein (CRP) levels and 7\% increase in interleukin-6 (IL-6) levels [37]. Chronic elevations in these proinflammatory cytokines have been shown to associate with an increased risk of diabetes [28] and therefore, habitual long sleep duration could alter the regulation of these cytokines and potentially affect glucose metabolism. Moreover, it is possible that long sleep duration might be a consequence of another unrecognized causative risk factor for insulin resistance. In addition, whether the lack of association between short sleep duration and insulin resistance was the results of a protective effect of vegetarian dietary patterns will need further investigations.

In addition to sleep duration, greater waist circumference and elevated levels of ALT were associated with insulin resistance. Waist circumference is considered as the best anthropometric indicator of abdominal visceral obesity [38] and has been used as a non-invasive method of identifying the presence of insulin resistance [39-41]. ALT, found primarily in the liver, is a specific indicator of heptocellular health. Several prospective studies have demonstrated elevated levels of ALT were an independent predictor of type 2 diabetes [42,43].

This study has some limitations that need to be considered. First, our study used cross-sectional design and therefore precluding direct inferences concerning temporal relationship of sleep duration and insulin resistance. Nevertheless, available evidence in diabetic studies indicated that disturbed sleep can impair glucose metabolism and poorly controlled glucose levels can also impair sleep [44]. Second, we have only measured sleep duration but not sleep quality. It is possible that poor

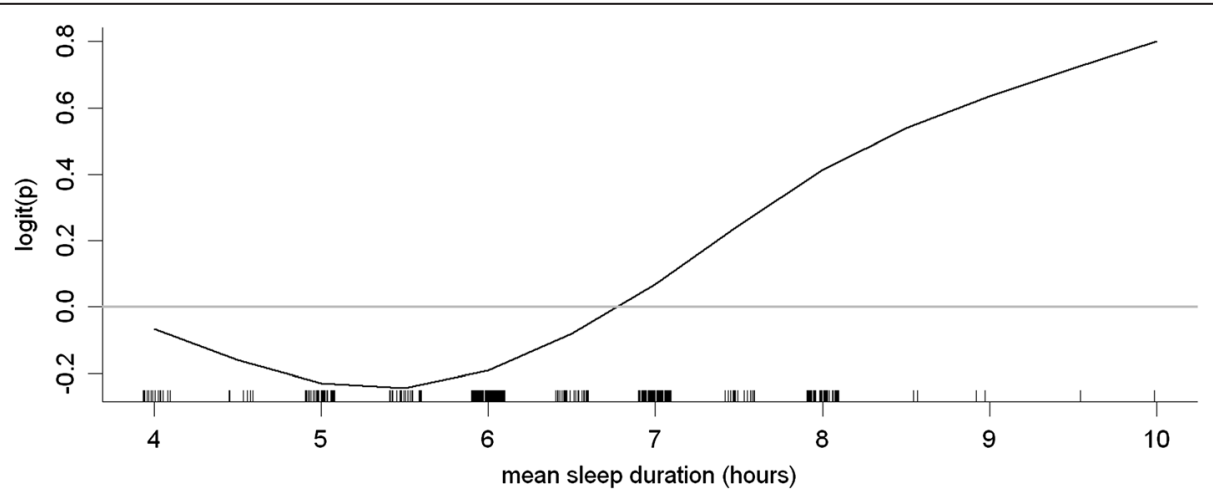

Figure 2 Plot of nonparametric smoothing from generalized additive model of sleep. Duration after adjusting for waist circumference and alanine transaminase levels. The $y$-axis is logit( $p$ ) where $p$ is the probability of insulin resistance. 
sleep quality, sleep apnea syndrome [45], and depression [46] can impact on insulin resistance. A more detailed questionnaire regarding sleep quality should be included in future studies. Third, information about sleep duration was based on participants' selfreport. Nevertheless, self-report have been shown to be a valid measurement of speed duration compared with polysomnography [47].

\section{Conclusions}

In conclusion, this study showed that longer duration of sleep was independently associated with insulin resistance in vegetarians, after controlling for potential confounders. Clinicians can use the codes provided in Additional file 1: Appendix 1 and readily available measurements of sleep duration, waist circumference, and ALT level as a simple adjunctive method for early detection of insulin resistance.

\section{Additional file}

Additional file 1: Appendix 1. Programming code in OpenOffice Calc Microsoft Excel, and R for calculating the probability of elevated insulin resistance (HOMA-IR >2) based on the multiple logistic regression.

\section{Abbreviations}

AIC: Akaike's Information Criterion; ALT: Alanine transaminase; AUC: Area under the curve; BUN: Blood urea nitrogen; ELISA: Enzyme Linked Immunosorbent Assay; HDL-C: High-density lipoprotein cholesterol; HOMA-IR: Homeostasis model assessment for insulin resistance; LDL-C: Low-density lipoprotein cholesterol; ROC: Receiver operating characteristic.

\section{Competing interests}

The authors declare that they have no competing interests.

\section{Authors' contributions}

JK Chiang conceived the research questions, designed the study and drafted the initial manuscript. M Koo was involved in the interpretation of the analysis and revision of the manuscript. JK Chang was involved in data analysis. YY Kao was involved in preparatory field works. All authors approve the final manuscript.

Institution at which the work was performed: Buddhist Dalin Tzu Chi General Hospital, Chiayi, Taiwan.

Grand support: Buddhist Dalin Tzu Chi General Hospital, Chiayi, Taiwan.

\section{Acknowledgements}

JK Chiang received research grants from Buddhist Dalin Tzu Chi General Hospital (DTCRD 96-13, 97-18, and 98 (2)-07).

\section{Author details}

'Department of Biotechnology, Chia Nan University of Pharmacy \& Science, Tainan, Taiwan. ${ }^{2}$ Dalla Lana School of Public Health, University of Toronto, Ontario, Canada. ${ }^{3}$ Department of Family Medicine, Buddhist Dalin Tzu Chi General Hospital, 2 Min-Sheng Road, Dalin, Chiayi, Taiwan.

Received: 4 April 2012 Accepted: 20 July 2012

Published: 16 August 2012

\section{References}

1. Wheaton AG, Perry GS, Chapman DP, McKnight-Eily LR, Presley-Cantrell LR, Croft JB: Relationship between body mass index and perceived insufficient sleep among U.S. adults: an analysis of 2008 BRFSS data. BMC Publ Health 2011, 11:295.
2. Shi Z, Taylor AW, Gill TK, Tuckerman J, Adams R, Martin J: Short sleep duration and obesity among Australian children. BMC Publ Health 2010, 10:609.

3. Van Cauter E, Spiegel K, Tasali E, Leproult R: Metabolic consequences of sleep and sleep loss. Sleep Med 2008, 9(Suppl 1):S23-S28.

4. Patel SR, Hu FB: Short sleep duration and weight gain: a systematic review. Obesity 2008, 16:643-653.

5. Kim J, Jo I: Age-dependent association between sleep duration and hypertension in the adult Korean population. Am J Hypertens 2010, 23:1286-1291.

6. Hoevenaar-Blom MP, Spijkerman AM, Kromhout D, van den Berg JF, Verschuren WM: Sleep duration and sleep quality in relation to 12-year cardiovascular disease incidence: the MORGEN study. Sleep 2011, 34:1487-1492

7. Thompson CL, Larkin EK, Patel S, Berger NA, Redline S, Li L: Short duration of sleep increases risk of colorectal adenoma. Cancer 2011, 117:841-847.

8. Koren D, Levitt Katz LE, Brar PC, Gallagher PR, Berkowitz RI, Brooks L: Sleep architecture and glucose and insulin homeostasis in obese adolescents. Diabetes Care 2011, 34:2442-2447.

9. Choi KM, Lee JS, Park HS, Baik SH, Choi DS, Kim SM: Relationship between sleep duration and the metabolic syndrome: Korean National Health and Nutrition Survey 2001. Int J Obes 2008, 32:1091-1097.

10. Chao CY, Wu JS, Yang YC, Shih CC, Wang RH, Lu FH, Chang CJ: Sleep duration is a potential risk factor for newly diagnosed type 2 diabetes mellitus. Metabolism 2011, 60:799-804.

11. Yaggi HK, Araujo AB, McKinlay JB: Sleep duration as a risk factor for the development of type 2 diabetes. Diabetes Care 2006, 29:657-661.

12. Cappuccio FP, D'Elia L, Strazzullo P, Miller MA: Sleep duration and all-cause mortality: a systematic review and meta-analysis of prospective studies. Sleep 2010, 33:585-592.

13. Liu R, Zee PC, Chervin RD, Arguelles LM, Birne J, Zhang S, Christoffel KK, Brickman WJ, Zimmerman D, Wang B, Wang G, Xu X, Wang X: Short sleep duration is associated with insulin resistance independent of adiposity in Chinese adult twins. Sleep Med 2011, 12:914-919.

14. Kobayashi D, Takahashi O, Deshpande GA, Shimbo T, Fukui T: Relation between metabolic syndrome and sleep duration in Japan: a large scale cross-sectional study. Intern Med 2011, 50:103-107.

15. Wang WS, Wahlqvist ML, Hsu CC, Chang HY, Chang WC, Chen CC: Ageand gender-specific population attributable risks of metabolic disorders on all-cause and cardiovascular mortality in Taiwan. BMC Publ Health 2012, 12:111.

16. Jennings JR, Muldoon MF, Hall M, Buysse DJ, Manuck SB: Self-reported sleep quality is associated with the metabolic syndrome. Sleep 2007, 30:219-223.

17. Hall MH, Muldoon MF, Jennings JR, Buysse DJ, Flory JD, Manuck SB: Selfreported sleep duration is associated with the metabolic syndrome in midlife adults. Sleep 2008, 31:635-643.

18. Reaven GM: Insulin resistance: the link between obesity and cardiovascular disease. Med Clin North Am 2011, 95:875-892.

19. Lakka HM, Laaksonen DE, Lakka TA, Niskanen LK, Kumpusalo E, Tuomilehto J, Salonen JT: The metabolic syndrome and total and cardiovascular disease mortality in middle-aged men. JAMA 2002, 288:2709-2716.

20. Kashyap SR, Defronzo RA: The insulin resistance syndrome: physiological considerations. Diab Vasc Dis Res 2007, 4:13-19.

21. Valachovicová M, Krajcovicová-Kudlácková M, Blazícek P, Babinská K: No evidence of insulin resistance in normal weight vegetarians. A case control study. Eur I Nutr 2006, 45:52-54.

22. Zhang $L$, Qin $L Q$, Liu AP, Wang PY: Prevalence of risk factors for cardiovascular disease and their associations with diet and physical activity in suburban Beijing, China. J Epidemio/ 2010, 20:237-243.

23. Rizzo NS, Sabaté J, Jaceldo-Siegl K, Fraser GE: Vegetarian dietary patterns are associated with a lower risk of metabolic syndrome: the adventist health study 2. Diabetes Care 2011, 34:1225-1227.

24. Fu CH, Yang CC, Lin CL, Kuo TB: Effects of long-term vegetarian diets on cardiovascular autonomic functions in healthy postmenopausal women. Am J Cardiol 2006, 97:380-383.

25. Wang YF, Chiu JS, Chuang MH, Chiu JE, Lin CL: Bone mineral density of vegetarian and non-vegetarian adults in Taiwan. Asia Pac J Clin Nutr 2008, 17:101-106.

26. Lu SC, Wu WH, Lee CA, Chou HF, Lee HR, Huang PC: LDL of Taiwanese vegetarians are less oxidizable than those of omnivores. J Nutr 2000, 130:1591-1596. 
27. Hung CJ, Huang PC, Li YH, Lu SC, Ho LT, Chou HF: Taiwanese vegetarians have higher insulin sensitivity than omnivores. Br J Nutr 2006, 95:129-135.

28. Patel SR, Zhu X, Storfer-Isser A, Mehra R, Jenny NS, Tracy R, Redline S: Sleep duration and biomarkers of inflammation. Sleep 2009, 32:200-204.

29. Chien MY, Wu YT, Kwan WK, Chien CL: Reliability of the Chinese version of the 7-day recall physical activity questionnaire and its correlation with impairments of disease and function. Formosan J Phys Ther 2003, 28:1-8.

30. Matthews DR, Hosker JP, Rudenski AS, Naylor BA, Treacher DF, Turner RC: Homeostasis model assessment: insulin resistance and beta-cell function from fasting plasma glucose and insulin concentrations in man. Diabetologia 1985, 28:412-419.

31. Balkau B, Charles MA, Drivsholm T, Borch-Johnsen K, Wareham N, Yudkin JS, Morris R, Zavaroni I, van Dam R, Feskins E, Gabriel R, Diet M, Nilsson P, Hedblad B: Frequency of the WHO metabolic syndrome in European cohorts, and an alternative definition of an insulin resistance syndrome. Diabetes Metab 2002, 28:364-366.

32. R 2.13.0. Development Core Team: $R$ : A language and environment for statistical computing. Vienna: R Foundation for Statistical Computing; 2011 http://www.R-project.org.

33. Knutson KL, Van Cauter E: Associations between sleep loss and increased risk of obesity and diabetes. Ann N Y Acad Sci 2008, 1129:287-304.

34. Spiegel K, Leproult R, Van Cauter E: Impact of sleep debt on metabolic and endocrine function. Lancet 1999, 354:1435-1439.

35. Patel SR, Malhotra A, Gottlieb DJ, White DP, Hu FB: Correlates of long sleep duration. Sleep 2006, 29:881-889.

36. Stranges S, Dorn JM, Shipley MJ, Kandala NB, Trevisan M, Miller MA, Donahue RP, Hovey KM, Ferrie JE, Marmot MG, Cappuccio FP: Correlates of short and long sleep duration: a cross-cultural comparison between the United Kingdom and the United States: the Whitehall II Study and the Western New York Health Study. Am J Epidemiol 2008, 168:1353-1364.

37. Pradhan AD, Manson JE, Rifai N, Buring JE, Ridker PM: C-reactive protein, interleukin 6, and risk of developing type 2 diabetes mellitus. JAMA 2001, 286:327-334.

38. Rankinen T, Kim SY, Pérusse L, Després JP, Bouchard C: The prediction of abdominal visceral fat level from body composition and anthropometry: ROC analysis. Int J Obes Relat Metab Disord 1999, 23:801-809.

39. Wahrenberg H, Hertel K, Leijonhufvud BM, Persson LG, Toft E, Arner P: Use of waist circumference to predict insulin resistance: retrospective study. BMJ 2005, 330:1363-1364.

40. Lee JM, Davis MM, Woolford SJ, Gurney JG: Waist circumference percentile thresholds for identifying adolescents with insulin resistance in clinical practice. Pediatr Diabetes 2009, 10:336-342.

41. Nilsson G, Hedberg P, Jonason T, Lonnberg I, Tenerz A, Forberg R, Ohrvik J: Waist circumference alone predicts insulin resistance as good as the metabolic syndrome in elderly women. Eur J Int Med 2008, 19:520-526.

42. Vozarova B, Stefan N, Lindsay RS, Saremi A, Pratley RE, Bogardus C, Tataranni PA: High alanine aminotransferase is associated with decreased hepatic insulin sensitivity and predicts the development of type 2 diabetes. Diabetes 2002, 51:1889-1895.

43. Sattar N, Scherbakova O, Ford I, O'Reilly DS, Stanley A, Forrest E, Macfarlane PW, Packard CJ, Cobbe SM, Shepherd J: Elevated alanine aminotransferase predicts new-onset type 2 diabetes independently of classical risk factors, metabolic syndrome, and C-reactive protein in the West of Scotland Coronary Prevention Study. Diabetes 2004, 53:2855-2860.

44. Martins RC, Andersen ML, Tufik S: The reciprocal interaction between sleep and type 2 diabetes mellitus: facts and perspectives. Braz J Med Biol Res 2008, 41:180-187.

45. Tasali E, Ip MS: Obstructive sleep apnea and metabolic syndrome: alterations in glucose metabolism and inflammation. Proc Am Thorac Soc 2008, 5:207-217.

46. Mclntyre RS, Soczynska JK, Konarski JZ, Woldeyohannes HO, Law CW, Miranda A, Fulgosi D, Kennedy SH: Should Depressive Syndromes Be Reclassified as "Metabolic Syndrome Type II"? Ann Clin Psychiatry 2007, 19:257-264.

47. Signal TL, Gale J, Gander PH: Sleep measurement in flight crew: comparing actigraphic and subjective estimates to polysomnography. Aviat Space Environ Med 2005, 76:1058-1063.

\section{doi:10.1186/1471-2458-12-666}

Cite this article as: Chang et al: Association of sleep duration and insulin resistance in Taiwanese vegetarians. BMC Public Health 2012 $12: 666$.

\section{Submit your next manuscript to BioMed Central and take full advantage of:}

- Convenient online submission

- Thorough peer review

- No space constraints or color figure charges

- Immediate publication on acceptance

- Inclusion in PubMed, CAS, Scopus and Google Scholar

- Research which is freely available for redistribution

Submit your manuscript at www.biomedcentral.com/submit 